\title{
Broadening Math Outreach
}

\section{Glen Whitney}

Note: The opinions expressed here are not necessarily those of Notices.

As I think anyone reading these Notices is aware, mathematics suffers from a tremendous image problem in this country. For large segments of the population, math is the subject we love to hate. People proclaim almost proudly that they "are terrible at math," something they would never do concerning other topics like reading or American history. We know the stereotypes: people who like math are out of touch, maladjusted, or just plain weird. People outside our profession are also confused about what mathematicians do; maybe work out the solutions to really tough calculus exercises?

The problem isn't only anecdotal. Just to scratch the surface of quantitative investigation into attitudes toward mathematics, I'll mention a couple of recent publications. In a 2017 national survey of teenagers by the Thomas B. Fordham Institute [3], one question asked respondents to choose their least favorite subject in school. Mathematics was the overwhelmingly most common answer, with 34\% of the respondents selecting it. A 2016 survey article by Dowker, Sarkar, and Looi [1] cites several studies that suggest "attitudes to mathematics tend to deteriorate with age during childhood and adolescence," as well as other studies that indicate that math anxiety affects variously some fraction of students ranging from $6 \%$ to $68 \%$ (depending on the definition of "math anxiety" and the population under consideration).

Glen Whitney is a visitor in the mathematics department at Harvard University. His email address is gwhi tney@post. harvard. edu.

For permission to reprint this article, please contact: reprint -permission@ams.org.

DOI: https://dx.doi.org/10.1090/noti 1860
Not only is this situation real, it's a problem that we as mathematicians should be concerned about. Some reasons are obvious: As technology advances, mathematical skills, interpreted broadly, become ever more important in the workplace, and we need to help foster a productive workforce of the future. With the AMS Annual Survey showing the fraction of PhDs awarded to Americans flat at slightly under $50 \%$, should the rate of foreign students leaving the country after graduate school rise, there could even be a problem replacing our profession's own ranks. Other reasons may be more indirect, but nevertheless warrant concern: Can we assume a reliable supply of public or even private funding from a society that fails to appreciate or even understand what mathematicians do? Most of us are in this profession because of the thrill and beauty of mathematical discovery; isn't there intrinsic value in sharing what we can of those experiences to as broad a population as possible?

Hence, there's a need for institutions dedicated to improving the public perception of mathematics. The image problems aren't going to solve themselves. Certainly, the commercial success of technology and financial firms based on their use of mathematics provides some good raw material for improving the status of mathematics, but someone has to be advocating on behalf of mathematics using that material. And I'm specifically interested in outreach directly to general audiences. Ideally, high-quality, engaging but demanding public education in mathematics would solve much of the problem. Working toward that goal is of course a good idea, and there exist many fine efforts in that direction, but there are entrenched reasons why public-education reform alone is unlikely to fix perception 
problems plaguing mathematics in at least the short-to-medium term. These reasons include the limitations imposed by the elementary and secondary canon of math topics, the nature of standardized testing, generally less stringent math-background requirements for elementary educators, and the economic interests of businesses involved in school education.

In the early 2000s, well before I had contemplated most of the above issues consciously, I had the opportunity to visit the Goudreau Museum of Mathematics in Art and Science. (At the time, to visit at all, the Goudreau required at least ten people to come there together, and the visit had to be by appointment.) Whatever the popular image of mathematics might be, here was a place where math was thoroughly celebrated, played with, and enjoyed. The museum consisted of just two converted classrooms in a Herricks, Long Island junior-high-turned-community-center, densely packed with games and puzzles; its ceilings burgeoned with a stunning array of polyhedral models. I remember thinking when leaving, "What a great country this is - there can be a museum about anything, even mathematics!" Tacitly, I was acknowledging to myself the fringe status of mathematics in American culture. In the following years, however, I became the "coach" of the after-school mathematics club at my local elementary school, and I began to reflect more consciously on math's social status.

Thus in 2008, when I learned of the closure of the Goudreau Museum, I conceived of a new math museum project on a larger scale and with an explicit mission to improve the public perception of mathematics. By mid-2009, the project dubbed "MoMath" had opened a traveling exhibtion, the Math Midway, and kicked off a capital campaign to fund the opening of a physical museum. In late 2012, that museum opened its doors in New York City. Along the way, MoMath organized or co-organized a long-running series of public lectures on mathematical topics, Math Encounters, a biannual conference on recreational mathematics, MOVES, and a biannual conference on math outreach, MATRIX (so far held just in Europe). My connection with MoMath ended in the fall of 2017, shortly before it celebrated its fifth anniversary open to the public. Despite its accomplishments and milestones, MoMath is not enough to resolve or even adequately address math's public perception problems. This is a big country, so naturally these are big problems, beyond the scope of any one institution.

So, what could improve math outreach further? There are numerous math blogs and video channels that undoubtedly reach a larger audience than it's possible for a single physical museum to do, so stronger ties and mutual support between those forms of communication and organizations focusing on math outreach could amplify the effectiveness of their efforts. Careful investigation of the impact of exhibits and programs, and creative experimentation with how they are presented, could help strengthen the link between what's presented and what the audience recognizes as "mathematics." All too often, visitors to math exhibits respond along the lines that "this is just a playground with numbers thrown in," not really mathematics.

Even when someone does connect an activity or program with "math," we need to provide more compelling narratives about those mathematical topics to win the hearts and minds of participants. We need to do a better job of connecting mathematics to peoples' lives and the world around them, while continuing to celebrate the value and beauty of pursuing mathematical ideas for their own sake. We need to show ways that mathematics has improved the human condition, and highlight its role in other accomplishments of our civilization. And to broaden the audience that we can connect with, we need to do a better job of humanizing mathematics and mathematicians. The stories of a wide range of mathematicians, especially contemporary practitioners, with whom diverse audiences can identify and whose passions they can come to understand, can be a powerful tool for engagement with mathematics.

So what are some elements of a broader effort to solve mathematics' image problem? First, we need more institutions dedicated to the effort. Some of these are or will be existing organizations. There's not space here to mention all of the existing institutions and programs I'm aware of, and I know there are many others that I've yet to have the pleasure of learning about. To help strengthen the network of math outreach efforts in this country, and avoid fragmentation of the community involved in such efforts, I have established a list of such programs (at studioinfinity.org/outreach). If you know of or participate in a program not mentioned here, please visit and submit your program to the list!

So, what are some notable efforts out there already? The Mathematical Sciences Research Institute (MSRI) is strengthening its outreach activities: In Spring 2019, it is organizing the third National Math Festival. Modeled after the collection of science festivals that arose in the US in the past decade, this festival in Washington, DC, will consist of a day of public speakers, temporary exhibits, and group activities celebrating the beauty and diversity of mathematics. MSRI also established the National Association of Math Circles, which fosters groups of interested students who meet periodically to creatively explore wide-ranging mathematical topics with the guidance of math professionals. The American Institute of Mathematics administers the Julia Robinson Mathematics Festivals, instigated by Nancy Blachman. These JRM Festivals occur in many sites around the country each year, and present a buffet of facilitated tabletop math activities carefully chosen for their power to engage a broad audience. The Association of Women in Mathematics organizes and sponsors Sonia Kovalevsky days, consisting of "a program of workshops, talks, and problem-solving competitions for female high school and middle school students and their teachers, both women and men." 
The US also needs more mathematics museums; if Germany can support ten, certainly there should be more than one in this country. Fortunately, some efforts are already underway. Frederic Mahieu is organizing a Math Cultural Center of Chicago (info@mathcul turalcenter . org). His vision is to "approach math through its culture, history and applications and share fascinating stories about math," but also to give visitors ample opportunities for "exploring all aspects of what math is: doing, thinking, observing, creating." There is another math museum project in Boston at an even earlier stage in development, and plenty of room for more such efforts around the country.

Second, we need to strengthen the math outreach activities at related institutions. Just based on membership in the Association of Science and Technology Centers, there are over 600 science museums in the country. Currently, only a small percentage mount significant mathematics exhibitions or have robust permanent exhibits celebrating mathematics in its own right. (Notable contributors to public math content include the Oregon Museum of Science and Industry, the Science Museum of Minnesota, the Boston Museum of Science, the New York Hall of Science, and the North Carolina Museum of Life and Science, among others.) Providing attractive, low-cost, high-visitor-engagement exhibit and programming options, and advocating for their use at science centers, would go a long way toward geographically broadening math outreach.

Also, American universities, colleges, and math departments could do more. Numerous universities in the United Kingdom have offices and/or directors of mathematics outreach (just try searching the phrase "UK university director of math outreach"); imagine the impact if a similar percentage of American higher educational institutions followed suit. (In fact, as White and Pantano pointed out a couple of years ago in these Notices [4], there's a great deal that the US could learn from-and contribute to-international math outreach, and certainly I applaud efforts toward such interchange.) There are already some efforts at US institutions along these lines: The STEAM Factory at OSU, organized by Jim Fowler and others, has created an interdisciplinary network that works together to make math and science accessible to general audiences in the Columbus, $\mathrm{OH}$, area. The Arizona Math Road Show by Bruce Bayly et al. at the University of Arizona has brought an entertaining and participatory performance-based math program to numerous schools and other venues, literally driving it across the country in a reconditioned school bus. Dwyer and Schovanec report on a variety of math outreach initiatives at Texas Tech in their 2013 Notices article [3]. I know there are others, and I hope you'll add them to the developing list on-line. But more such initiatives are needed, and they can make the "broader impact" efforts required by many granting agencies significantly more meaningful and effective.
Third, we need additional types of activities and delivery methods, to broaden the audience. Participants need to see people they identify with as role models presenting the material; they need to see peers excited about mathematics. Demographic studies show that visitors to science museums are significantly less racially and economically diverse than the general population. So we need to take math outreach to places where we can reach those who are not coming to science centers: to visit schools and after-school programs, or even to stadiums, malls, or basketball courts. (Certainly there already exist programs designed to reach diverse populations. One example is the Art of Problem Solving's Bridge to Enter Advanced Mathematics headed by Dan Zaharopol, which identifies groups of promising middle schoolers from underrepresented populations in New York and Los Angeles and provides summer programs highlighting the thrill of problem-solving and the breadth of mathematics, along with ongoing support through college applications. But more programs that expand the population who can become engaged with mathematics are needed. It's also important to broaden the age range we reach; for example, the Bedtime Math enterprise started by Laura Overdeck provides books, math club kits, and other materials that can foster positive attitudes toward math for 3-8 year olds. There's definitely room for programs tailored to older Americans as well. And finally, we need effective methods to measure the impact of all these efforts; it's nearly impossible to optimize what you can't measure (or aren't measuring).

All of these enterprises need the involvement of professional mathematicians. The science festival movement mentioned above provides many opportunities for practicing scientists to meet with general audiences and talk about or show off what they do. But in my conversations with science festival staff, they express disappointment at the number of mathematicians who respond to calls for participation, as compared to biologists, chemists, physicists, etc. Partly as a result, most science festivals have little mathematics content. And presuming that we can increase the number of math museums and other math-outreach organizations, all of those institutions will need advisors, idea generators and contributors, and fact-checkers. (Let's make sure to get the math right when we're using it for outreach!) So keep your eyes open for opportunities to help with math outreach and get involved-you'll find, as I have, that it's an exciting, challenging, and fundamentally rewarding pursuit. 


\section{References}

[1] Dowker A, Sarkar A, Looi CY, Mathematics Anxiety: What Have We Learned in 60 Years? Frontiers In Psychology, Vol. 7, 2016, p. 508. (doi:10.3389/fpsyg.2016.00508)

[2] Dwyer J, Schovanec L, Revisiting an Outreach Mathematician, Noti. Amer. Math. Soc, Vol. 60, No. 7, 2013, pp. 924926. (doi:10.1090/noti1023)

[3] Geraci J, Palmerini M, Cirillo P, and McDougald M, "What Teens Want From Their Schools: A National Survey of High School Student Engagement," Thomas B. Fordham Institute, 2017. (edexcellence.net/publications /what-teens-want-from-their-schools)

[4] White D, Pantano A, et. al., Joining Forces in International Mathematics Outreach Efforts, Notices of the American Mathematical Society, Vol. 63, No. 9, 2015, pp. 1042-1049. (doi:10.1090/noti1422)

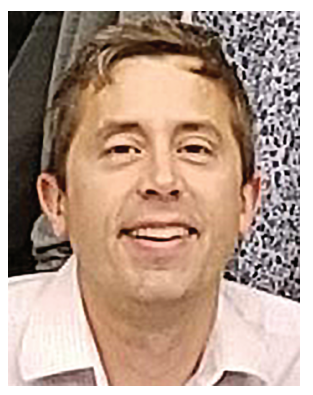

Glen Whitney

Credits

Author photo is courtesy of Glen Whitney.

Co-managed by the AMS and the MAA for many years, the Joint Mathematics Meetings (JMM) is the world's largest gathering of mathematicians.

Starting in 2022, the AMS will manage the JMM solely, while the MAA and other organizations will continue to participate.

This is a chance for us to reimagine the JMM to ensure a continued rich experience, and to ask you to share your ideas for the JMM in 2022 and beyond. How would you rethink or improve the meetings?

Thank you for helping to ensure that the JMM continues to offer "something for everyone" within the mathematical community.

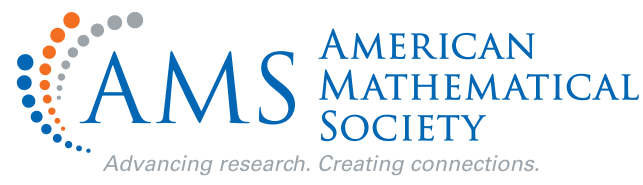

